\title{
Can we "REFINE" the art of predicting ischemia on SPECT myocardial perfusion imaging?
}

\author{
Karthik Ananthasubramaniam, MD, FACC, FASE, FASNC, FSCCT, FSCMR, FRCP, ${ }^{a}$ \\ and Parthiban Arumugam, MBBS ${ }^{\mathrm{b}}$ \\ a Heart and Vascular Institute, Henry Ford West Bloomfield Hospital, West Bloomfield, MI \\ b Department of Nuclear Medicine, Manchester University, Manchester, United Kingdom
}

Received Nov 6, 2021; accepted Nov 7, 2021

doi: $10.1007 / \mathrm{s} 12350-021-02867-5$

\section{See related article, pp. 3221-3232}

Myocardial Perfusion Imaging (MPI) with Single Photon Emission Computed Tomography (SPECT) continues to be one of the most commonly performed non-invasive functional imaging modality for patients with stable suspected cardiac chest pain ${ }^{1}$. Worldwide 20 million SPECT studies performed and, in the US alone approximately 6.5 million studies are done. While MPI SPECT has high diagnostic accuracy and excellent prognostic value, recent trends regarding the 'yield' of SPECT in terms of identifying patients with significant coronary artery disease (CAD) requiring appropriate medical or revascularisation strategy has been $\operatorname{low}^{2-4}$. An important contributor to this could be increasing selection of low pre test likelihood patients for stress testing based on existing CAD risk estimators. ${ }^{5,6}$ Other reasons for the low " yield" include referral bias, testing asymptomatic patients and use of medical therapy for slowing down atherosclerosis progression leading to lower frequency of abnormal scans. In a large single study of SPECT studies from 1991 to 2009 a marked decline in overall abnormal SPECT studies was noted (from $40.9 \%$ in 1991 to $8.7 \%$ in 2009) along with marked decline in ischemic SPECT (from 29.6\% in 1991 to $5 \%$ in 2009). ${ }^{7}$ These trends have brought to

Dr Ananthasubramaniam receives research Grant from Astellas Pharma and is on advisory panel for Alnylam Pharmaceuticals

Reprint requests: Karthik Ananthasubramaniam, MD, FACC, FASE, FASNC, FSCCT, FSCMR, FRCP, Heart and Vascular Institute, Henry Ford West Bloomfield Hospital, 6777 W Maple, West Bloomfield, MI 48322; kananth1@hfhs.org

J Nucl Cardiol 2022;29:3233-5.

$1071-3581 / \$ 34.00$

Copyright (C) 2021 The Author(s) under exclusive licence to American Society of Nuclear Cardiology attention that the nuclear cardiology world needs to rethink strategies of how best to utilize SPECT in the right patient and right clinical setting. This is key to maintain the value of SPECT as being the " gate keeper' for further invasive testing when indicated.

The existing pre-test probability (PTP) estimates are easy to use as information is readily available to the referrer: age, sex and angina characteristics (DiamondForrester). The downside of PTP is they were formulated back in 1970s using coronary angiography and are not representative of the chest pain population seen in daily practice currently. Hence, they consistently tend to overestimate likelihood of CAD. To address this problem, new modified Diamond and Forrester estimation and new societal guidelines have been published. ${ }^{8-10}$ However even with the modifications, the overestimation issue still persists as evident in studies evaluating the newer models. ${ }^{4,11}$ In a dramatic change of approach to evaluation of chest pain the United Kingdom National Institute of Clinical Excellence (NICE) issued an update on it original guideline (2010) in 2016 eliminating PTP and just using clinical judgement if chest pain is typical or atypical for CAD. ${ }^{12}$

Thus, there continues to be a need for more efficient ways to predict an abnormal SPECT scan given significant strain on resources for healthcare service providers, scrutiny on medical imaging and emergence of competing modalities such as coronary computed tomography.

In this backdrop the REFINE SPECT investigators present their analysis in current issue of Journal of Nuclear Cardiology, where they leverage the data from a large multicentre registry. Their primary aim was to validate findings from a prior single centre study which showed a strong inverse relationship between left ventricular ejection fraction and scar tissue to the extent of ischemia. ${ }^{13}$ In that large single center study of over 27,000 patients the frequency of SPECT ischemia was 
only 6.6\% among patients with an LVEF $>55 \%$ but $38.1 \%$ for patients with LVEF $<45 \%(\mathrm{P}<0.001)$. The frequency of myocardial ischemia was also fourfold higher among patients with known CAD vs no known CAD $(28.0 \%$ vs $6.5 \%, \mathrm{P}<0.001)$ and approximately threefold higher among patients with typical angina vs patients with atypical symptoms. Thus REFINE SPECT registry was used to see if such findings were reproducible in a large multicenter registry.

The REFINE SPECT international registry comprises a large numbers of SPECT scans done with high efficiency SPECT cameras and contains a rich repository of clinical, demographic, stress test and SPECT variables. ${ }^{14}$ The main goal of this registry was to establish a large SPECT dataset and use machine learning methods to integrate quantitative date to " pre scan " clinical variables. The investigators have previously published their experience from this registry ${ }^{15}$ showing that even minimal defects deciphered with quantitative analysis despite normal visual reads could carry prognostic significance.

In the present study, ${ }^{16} 19,690$ patients from five centres with a core lab quantitative analysis of MPI SPECT obtained using ultrafast solid state detector technology has confirmed that key and well established patient demographics (increasing age, known CAD and male gender) as important predictors of ischaemia on SPECT MPI in addition to scan related findings. Also, the investigators confirm their single center study finding of an inverse relationship between $L V$ ejection fraction and resting total perfusion defect (TPD) to prevalence of ischemia. Surprisingly comparing the different symptoms subgroups (typical angina, atypical angina, non-cardiac chest pain and asymptomatic), using multivariate analysis, the authors reported no difference in predictors of ischaemia. Additionally, prevalence of ischemia was in the following order based on symptoms: typical angina, asymptomatic patients, atypical and non-cardiac chest pain. In clinical practice one would envisage using predictors of ischemia to not test particularly the asymptomatic and non-cardiac chest pain groups; but the findings of this study do not help delineate this important aspect.

A reassuring aspect of predictability of ischemic SPECT scans shown in this study is that many currently used clinical risk markers such as age, male gender, typical angina, pharmacologic stress, known CAD, cardiac risk factors were all associated with abnormal scans. As shown in Table 4 of this study the top 5 rank order of predictors of abnormal SPECT in patients with no known CAD included male gender, lower LV EF, \% myocardial scar, increasing age and typical angina. While most of this information is readily available and can be giving weightage for predicting ischemia before ordering tests, the study findings of scar extent and LV EF as strong predictors of ischemia are not typically available in patients prior to SPECT to use for predicting ischemia. One could potentially tap into pre-existing information like prior SPECT or echocardiographic ejection fraction and extent of visually reported scar if available as an add on to above factors but this not always available before SPECT. Also in daily clinical practice it is more important to known which patients without known CAD could likely benefit from SPECT rather than those with established CAD where SPECT is done for either assessment of residual ischemia or prognostication.

Although the REFINE SPECT registry helps confirm the relationship between EF, scar and ischemia, significant limitations exist (appropriately acknowledged by the authors) in this study apart from those discussed above. This study population is notable for significant heterogeneity of the patient population and varying local prevalence of CAD at different centers (supplemental Table 2 of study) and is limited due to its inherent retrospective design with 'unadjusted' patient population in terms of referral bias and unknown confounding factors. Also as outlined by authors this study was not designed to" a priori'" develop a predicting algorithm for identifying ischemia on SPECT. It is sobering to see the large number of "asymptomatic or non-anginal" chest pain patients (50-70\%) undergoing SPECT studies across the range of ischemic studies. (supplemental Table 3). No appropriate use criteria was used for test selection in this real world registry. Although the exact reasons for testing asymptomatic patients is not stated in this study, is a time for reflection for clinicians using SPECT to really take a close look at how we utilize this technology. Leading cardiac societal recommendations and randomized trials clearly outline that testing asymptomatic patients is not associated with improved outcomes and is discouraged even if significant ischemia is detected in a small proportion of patients. Hopefully as the registry continues to evolve and machine learning tools are applied, we can glean further data to be able to identify predictors of ischemia on SPECT better for appropriate indications and in the right patient population.

\section{Disclosure}

The authors have indicated that they have no financial conflict of interest.

\section{References}

1. Hachamovitch R, Hayes SW, et al. Stress myocardial perfusion single-photon emission computed tomography is clinically effective and cost effective in risk stratification of patients with a high likelihood of coronary artery disease (CAD) but no known CAD. J Am Coll Cardiol 2004;43:200-8. 
2. Patel MR, Peterson ED, et al. Low diagnostic yield of elective coronary angiography. N Engl J Med 2010;362:886-95. https://doi. org/10.1056/NEJMoa0907272.

3. Cheng VY, Berman DS, et al. Performance of the traditional age, sex, and angina typicality-based approach for estimating pretest probability of angiographically significant coronary artery disease in patients undergoing coronary computed tomographic angiography: results from the multinational coronary CT angiography evaluation for clinical outcomes: an international multicenter registry (CONFIRM). Circulation 2011;124:2423-32. https://doi. org/10.1161/CIRCULATIONAHA.111.0392.

4. Batal O, Malhotra S, et al. Performance of traditional pretest probability estimates in stable patients undergoing myocardial perfusion imaging. Circ Cardiovasc Imaging 2019;12:e008473. h ttps://doi.org/10.1161/CIRCIMAGING.118.008473.

5. Diamond GA, Forrester JS. Analysis of probability as an aid in the clinical diagnosis of coronary-artery disease. $\mathrm{N}$ Engl J Med 1979;300:1350-8. https://doi.org/10.1056/ NEJM197906143002402

6. Chaitman BR, Bourassa MG, et al. Angiographic prevalence of high-risk coronary artery disease in patient subsets (CASS). Circulation 1981;64:360-7. https://doi.org/10.1161/01.cir.64.2.360.

7. Rozanski Am Granser $\mathrm{H}$, et al. Temporal trends in inducible myocardial ischemia during cardiac stress testing 1991-2009. J Am Coll Cardiol 2013;61:1054-65.

8. Montalescot G, Sechtem U, Document Reviewers, ESC Committee for Practice Guidelines, Task Force Members, et al. ESC guidelines on the management of stable coronary artery disease: the Task Force on the management of stable coronary artery disease of the European Society of Cardiology. Eur Heart J 2013;2013:2949-3003. https://doi.org/10.1093/eurheartj/eht296.

9. Genders TS, Steyerberg EW, CAD Consortium, et al. A clinical prediction rule for the diagnosis of coronary artery disease: validation, updating, and extension. Eur Heart J 2011;32:1316-30. h ttps://doi.org/10.1093/eurheartj/ehr014.

10. Wolk MJ, Bailey SR et al.; American College of Cardiology Foundation appropriate use criteria task force ACCF/AHA/ASE/ ASNC/HFSA/HRS/SCAI/SCCT/SCMR/STS 2013 multimodality appropriate use criteria for the detection and risk assessment of stable ischemic heart disease: a report of the American College of Cardiology Foundation Appropriate Use Criteria Task Force, American Heart Association, American Society of Echocardiography, American Society of Nuclear Cardiology, Heart Failure Society of America, Heart Rhythm Society, Society for Cardiovascular Angiography and Interventions, Society of Cardiovascular Computed Tomography, Society for Cardiovascular Magnetic Resonance, and Society of Thoracic Surgeons. J Am Coll Cardiol. 2014; 63:380-406. https://doi.org/10.1016/j.jacc. 2013.11.009

11. Greenwood JP, Ripley DP, CE-MARC 2 Investigators, et al. Effect of care guided by cardiovascular magnetic resonance, myocardial perfusion scintigraphy, or NICE guidelines on subsequent unnecessary angiography rates: the CE-MARC 2 randomized clinical trial. JAMA 2016;316:1051-60. https://doi.org/10.1001/jama.201 6.12680 .

12. Recent-onset chest pain of suspected cardiac origin: assessment and diagnosis Clinical guideline [CG95] Published: 24 March 2010 Last updated: 30 November 2016. https://www.nice.org.uk/g uidance/CG95

13. Rozanski A, Miller RJH, Han D, et al. Prevalence and predictors of inducible myocardial ischemia among patients referred for radionuclide stress. J Nucl Cardiol (Journal publication details pending)

14. Slomka PJ, Betancur J, et al. Rationale and design of the REgistry of Fast Myocardial Perfusion Imaging with NExt generation SPECT (REFINE SPECT). J Nucl Cardiol 2020;27:1010-21. h ttps://doi.org/10.1007/s12350-018-1326-4.

15. Yuka O, Julian B, et al. 5-Year prognostic value of quantitative versus visual MPI in subtle perfusion defects: results from REFINE SPECT. J Am Coll Cardiol Img 2020;13:774-85.

16. Han D, Rozanski A, Miller R, et al. Prevalence and predictors of automatically quantified ischemia within a multicenter international registry. J Nucl Cardiol 2021. https://doi.org/10.1007/ s12350-021-02829-x.

Publisher's Note Springer Nature remains neutral with regard to jurisdictional claims in published maps and institutional affiliations. 